doi: $10.15407 /$ ujpe61.05.0393

Y. ACHENEFE, T. SENBETA, V. N. MAL'NEV

Department of Physics, Addis Ababa University

(P.O.Box 1176, Addis Ababa, Ethiopia; e-mail: y.achenefe@yahoo.com)

\title{
ELECTRON SCATTERING
} PACS 75.50.-xx, 75.75.-c IN GRAPHENE BY REMOTE NANOMAGNETS

\begin{abstract}
The elastic electron scattering by a nonuniform magnetic field of remote nanomagnets in graphene is considered with the help of a modified Born approximation. The nanomagnets are modeled by point-like magnetic dipoles oriented transversally and in parallel to the graphene plane. They can form rather high magnetic fields without any damage of the graphene plane. The electron scattering cross sections are obtained in the closed form and analyzed numerically. It is shown that this mechanism of scattering has nonzero backscattering crosssection and can considerably affect the graphene conductivity.
\end{abstract}

Keywords: graphene, nanomagnets, scattering amplitude.

\section{Introduction}

It is known that the electron scattering by charged impurities in graphene is dominant over all other mechanisms scattering, but does not considerably affects the very high mobility of charge carriers in graphene [1]. The electron scattering in graphene by impurities with electric and magnetic dipoles has been studied in [2] with the help of a modified Born approximation. It was shown that the nonsymmetric scattering potential of these impurities causes the nonzero backscattering cross-sections. The scattering effects of the magnetic dipoles is negligible due to the comparative smallness of the magnetic moments of natural atoms and molecules.

Modern technology enables one to manufacture nanomagnets with anomalous magnetic moments that can be more than 10 Bohr magnetons per atom of a nanomagnet [3-4]. In this paper, we consider the elastic electron scattering in graphene by the nonhomogeneous magnetic field of remote nanomagnets with gigantic magnetic moments within the Born approximation. The remote nanomagnets can support

(C) Y. ACHENEFE, T. SENBETA, V. N. MAL'NEV, 2016

ISSN 2071-0194. Ukr. J. Phys. 2016. Vol. 61, No. 5 the graphene plane or can be suspended above it and produce no deformations. The magnetic field of these nanomagnets is modeled by point dipoles and can be rather large up to $0.1 T$. The nanomagnets with gigantic magnetic moments considerably affect transport properties of electrons in graphene.

The paper is tailored as follows. In the second section, we formulate the problem and present the main equations. The third and fourth sections are devoted to obtaining the electron scattering cross-sections by remote magnetic dipoles perpendicular and parallel to the graphene plane and their numerical simulation. The conclusion summarizes the results of the paper.

\section{Main Equations}

The Schrödinger equation for the massless electron of graphene in an external magnetic field can be written as

$v_{\mathrm{F}} \hat{\sigma} \cdot\left(\hat{p}-\frac{e}{c} \mathbf{A}\right)\left(\begin{array}{l}\psi_{1} \\ \psi_{2}\end{array}\right)=E\left(\begin{array}{l}\psi_{1} \\ \psi_{2}\end{array}\right)$

where $v_{\mathrm{F}}$ is the Fermi velocity, $\hat{\sigma}=\left(\hat{\sigma}_{x}, \hat{\sigma}_{y}\right)$ are the Pauli matrices, $\hat{p}$ is the $2 \mathrm{D}$ momentum operator, $\mathbf{A}$ is 


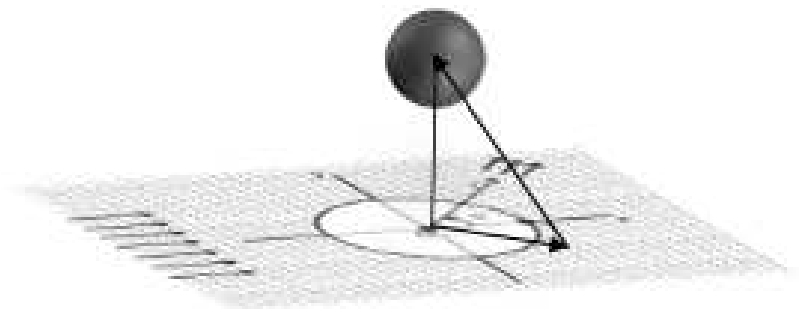

Fig. 1. Schematic diagram of a point-like magnetic dipole of a nanomagnet suspended above the graphene plane

the vector potential; $e$ and $c$ are the electron charge and the speed of light in vacuum, respectively. The functions $\psi_{1}$ and $\psi_{2}$ describe the electron in states related to the two sublattices of graphene and satisfy to the following coupled equations

$v_{\mathrm{F}} \hat{\pi}_{+} \psi_{1}=E \psi_{2}$,

$v_{\mathrm{F}} \hat{\pi}_{-} \psi_{2}=E \psi_{1}$,

where $\hat{\pi}_{ \pm}=\hat{p}_{ \pm}-e A_{ \pm} / c, \hat{p}_{ \pm}=\hat{p}_{x} \pm i \hat{p}_{y}, A_{ \pm}=$ $=A_{x} \pm i A_{y}$, where $\hat{p}_{x}, \hat{p}_{y}$ and $A_{x}, A_{y}$ are the components of the momentum operator and the vector potential, respectively, and $E$ is the electron energy.

Substituting $\psi_{2}$ from the first equation in (2) into the second one and vice versa, we obtain the following independent Helmholtz-type equations for $\psi_{1}$ and $\psi_{2}$ :

$\left(\nabla^{2}+k^{2}\right) \psi_{1,2}=\hat{V}_{1,2} \psi_{1,2}$.

Here, we introduced the wavevector $k=E /\left(\hbar v_{\mathrm{F}}\right)$ and the operator

$\hat{V}_{1,2}=\frac{1}{\hbar^{2}}\left\{\frac{e^{2}}{c^{2}} A^{2}-\frac{e}{c}\left[2 \mathbf{A} \cdot \hat{p} \pm \hbar B_{z}\right]\right\}$.

At large distances from the scattering center, the lefthand side of (4) is small, which allows us to seek its solution in the form

$\psi_{1,2}=\psi_{1,2}^{(0)}+\psi_{1,2}^{(1)}, \quad \psi_{1,2}^{(1)} \ll \psi_{1,2}^{(0)}$.

The wave functions $\psi_{1,2}^{(0)}$ describe the incident wave and satisfy the homogeneous equation (3). We chose them in the form of plane waves propagating along the $x$-axis:

$\psi_{1,2}^{(0)}=\frac{1}{\sqrt{2}} \exp (i k x)$.

The factor $1 / \sqrt{2}$ provides the unit normalization of the incident wave function

$\psi_{\text {inc }}=\frac{1}{\sqrt{2}}\left(\begin{array}{l}1 \\ 1\end{array}\right) \exp (i k x)$.
Substituting (5) in (3) with regard for (6) and the explicit form of operator (4), we obtain the equations for the scattered wave functions $\psi_{1,2}^{(1)}$ :

$\left(\nabla^{2}+k^{2}\right) \psi_{1,2}^{(1)}=V_{1,2} \psi_{1,2}^{(0)}$.

Here,

$V_{1,2}=\frac{1}{\hbar^{2}}\left\{\frac{e^{2}}{c^{2}} A^{2}-\frac{e \hbar}{c}\left[2 A_{x} k \pm B_{z}\right]\right\}$.

The scattered wave functions $\psi_{1,2}^{(1)}$ at large distances from the scattering center have the form

$\psi_{1,2}^{(1)}=f_{1,2}(\varphi) \frac{\exp (i k r)}{\sqrt{r}}$,

where $f_{1,2}(\varphi)$ are the scattering amplitudes, and $\varphi$ is the scattering angle. The asymptotic solutions of (8) allows one to single out the scattering amplitudes from the solutions of (10) [5]:

$f_{1,2}(\varphi)=-\frac{1}{4 \sqrt{\pi k}} \int \exp (-i \mathbf{q} \cdot \mathbf{r}) V_{1,2}(r) d^{2} r$

where $\mathbf{q}$ is a wave vector transferred to the electron under the elastic scattering $q=2 k \sin \varphi / 2$. The differential scattering cross-section is given by [2]

$\frac{d \sigma(\varphi)}{d \varphi}=2\left|f_{1}^{*}(\varphi) f_{2}(\varphi)\right|=2\left|f_{1}(\varphi)\right|\left|f_{2}(\varphi)\right|$.

Next, we consider the elastic electron scattering in graphene by the nonuniform magnetic field of a remote nanomagnet. Let us find the scattering amplitudes $f_{1,2}(\varphi)$ according to (11) and (9). The components of $\mathbf{A}$ and $B_{z}$ enter into $V_{1,2}(9)$. Let us model the nanomagnet by a sphere of radius $a$ with the built-in point magnetic dipole $\mathbf{d}$. Let it be located at the point $(0,0, l \geq a)$ above the graphene plane $(x, y, 0)$ (see Fig. 1). The vector potential and the corresponding magnetic field of the dipole can be obtained with the help of the known formulas

$\mathbf{A}=\frac{\mathbf{d} \times \mathbf{R}}{R^{3}}, \quad \mathbf{B}=\frac{3(\mathbf{d} \cdot \mathbf{R}) \mathbf{R}-R^{2} \mathbf{d}}{R^{5}}$.

Here, $R$ is a distance from the dipole to the observation point. To find the vector potential and the magnetic field in the graphene plane, one has to specify the orientation of the magnetic dipole $\mathbf{d}$ with respect to the graphene plane and to set $z=l$ in (13). Below, 
we consider the magnetic dipoles transversal and parallel to the graphene plane. It will be convenient to use the dimensionless coordinates and measure the distance in units $l$. For a vector of the dipole transverse to the graphene plane $\mathbf{d}=(0,0, d)$, we get the following components of $\mathbf{A}$ and $B_{z}$ from (13):

$$
\begin{aligned}
& A_{x}=-\frac{d}{l^{2}} \frac{y}{\bar{R}^{3}}, \quad A_{y}=\frac{d}{l^{2}} \frac{x}{\bar{R}^{3}}, \quad A_{z}=0, \\
& B_{z}=\frac{d}{l^{3}} \frac{2-r^{2}}{\bar{R}^{5}} .
\end{aligned}
$$

If the magnetic moment is parallel to the graphene plane, we get

$$
\begin{aligned}
& A_{x}=\frac{d}{l^{2}} \frac{\sin \alpha}{\bar{R}^{3}}, \quad A_{y}=-\frac{d}{l^{2}} \frac{\cos \alpha}{\bar{R}^{3}}, \\
& A_{z}=\frac{d}{l^{2}} \frac{y \cos \alpha-x \sin \alpha}{\bar{R}^{3}} \\
& B_{z}=3 \frac{d}{l^{3}} \frac{x \cos \alpha+y \sin \alpha}{\bar{R}^{5}} .
\end{aligned}
$$

Here, $\alpha$ is the angle between $\mathbf{d}$ and the $x$-axis, $\bar{R}=$ $=\sqrt{1+r^{2}}$ is the dimensionless distance from the dipole to a point of the graphene plane, $r^{2}=x^{2}+y^{2}$, with $x$ and $y$ being the dimensionless coordinates in the graphene plane. The distance $R$ is related to $\bar{R}$ as $R=l \bar{R}$.

\section{Scattering by a Magnetic Dipole Transverse to the Graphene Plane}

To find the scattering amplitudes $f_{1,2}(\varphi)$ according to (11), we have to substitute $\mathbf{A}$ and $B_{z}$ given by (14) in (9). In the accepted dimensionless coordinates, we get

$V_{1,2}=\frac{\lambda}{\bar{R}^{3}}\left\{\frac{\lambda r^{2}}{\bar{R}^{3}}+\left[2 \bar{k} y \mp \frac{2-r^{2}}{\bar{R}^{2}}\right]\right\}$,

where $\bar{k}=k l$ is the dimensionless wave vector of the electron, and

$\lambda=e d /(c \hbar l)$

is the dimensionless coupling constant. Let us estimate its numerical value. The magnetic moment of a spherical nanomagnet with radius $a$ is

$d=\frac{4 \pi}{3} \mu_{\mathrm{B}} a^{3} n N$,

where $N$ is a number of Bohr magnetons $\mu_{\mathrm{B}}$ per one atom of the nanomagnet material, and $n$ is its density number. Taking the numerical value $\mu_{\mathrm{B}}=$ $=10^{-20} \mathrm{Gs} \mathrm{cm}^{3}$ and $n=10^{22}$, we get the evaluation

$\lambda \sim 7 \times 10^{-5} N \frac{a}{l} a^{2}$,

where $a$ and $l$ must be in nanometers. It is reported that $10<N<100$ [3-4]. If we take $N=10$, then, for $a=10 \mathrm{~nm}$, nanomagnets are suspended over the graphene plane at a distance of the same order, $\lambda \sim 10^{-2}$. In this case, we can neglect the term $\sim \lambda^{2}$ in (16). But, for "larger" nanomagnets $40 \leq a \leq 10^{2} \mathrm{~nm}$, we can easily get $0.1 \leq \lambda \leq 1$. In this case, the term $\sim A^{2}$ in (9) must be taken into account. One more possibility to increase $\lambda$ is to arrange nanomagnets from the both sides of the graphene plane. The maximum magnetic field created by a nanomagnet according to (14), (15), and (18) is

$B \sim \mu_{\mathrm{B}} \frac{a^{3}}{l^{3}} n N$

This formula shows that the magnetic field of a remote magnetic dipole rapidly decreases with $l$. For $l \sim a$ and the numerical values of the parameters in (20) as above, we obtain that $B \sim 10^{3} \mathrm{Gs}=0.1 T$. Now, we calculate the scattering amplitudes $f_{1,2}(\varphi)$ in the case under consideration. Let us substitute (16) into (11), which we rewrite in the dimensionless coordinates. We obtain

$$
\begin{aligned}
& f_{1,2}(\varphi)=-\frac{\lambda}{4} \sqrt{\frac{l}{\pi \bar{k}}} \int_{0}^{\infty} d r^{\prime} \int_{0}^{2 \pi} d \phi^{\prime} \exp \left(-i \bar{q} r^{\prime} \cos \phi^{\prime}\right) \times \\
& \times\left\{\frac{\lambda r^{\prime 3}}{R^{\prime 6}}+2 \frac{\bar{k} r^{\prime 2} \cos \left(\phi^{\prime}-\varphi / 2\right)}{R^{\prime 3}} \mp \frac{2 r^{\prime}-r^{\prime 3}}{R^{\prime 5}}\right\} .
\end{aligned}
$$

Here, $R^{\prime}=\sqrt{1+r^{\prime 2}}$, and $\bar{q}=2 \bar{k} \sin \varphi / 2$ is the dimensionless transferred wavevector. The integration in $(21)$ is carried out in the coordinate system with the $y^{\prime}$ along the vector $\overline{\mathbf{q}}$, where $\overline{\mathbf{q}} \cdot \mathbf{r}^{\prime}=\bar{q} r^{\prime} \cos \phi^{\prime}$. It is obtained by the anticlockwise rotation of the initial coordinate system $x, y$ by the angle $\varphi / 2$. In the integrand, we substitute $y^{\prime}=r^{\prime} \cos \left(\phi^{\prime}-\varphi / 2\right)$. The integration over $\phi^{\prime}$ gives the Bessel functions according to the known relation [6]

$$
\int_{0}^{2 \pi} d \phi^{\prime} \exp \left[i\left(z \cos \phi^{\prime}+n \phi^{\prime}\right)\right]=2 i^{n} \pi J_{n}(z),
$$


where $J_{n}(z)$ is the Bessel function with an integer $n$. While integrating (21) over $r^{\prime}$, we obtain the integrals

$I_{n m}^{(p)}(q)=\int_{0}^{\infty} J_{n}(q r) \frac{r^{p} d r}{\left(1+r^{2}\right)^{m / 2}}$

They can be calculated in the closed form (see Appendix). Presenting the numerator of (23) as $r^{p}=$ $=\left(1+r^{2}-1\right) r^{p-2}$, we can obtain

$I_{n m}^{(p)}(\bar{q})=I_{n m-2}^{(p-2)}(\bar{q})-I_{n m}^{(p-2)}$.

With the help of (23) and (24), we present the scattering amplitudes (21) as

$f_{1,2}(\varphi)=-\frac{\lambda}{2} \sqrt{\frac{\pi l}{\bar{k}}}\left\{I_{ \pm}(\bar{q})+2 i \bar{k} I_{13}^{(2)}(\bar{q}) \cos \varphi / 2\right\}$,

where $I_{ \pm}(\bar{q})=\lambda I_{06}^{(3)}(\bar{q}) \pm\left[2 I_{05}^{(1)}(\bar{q})-I_{05}^{(3)}(\bar{q})\right]$.

In view of (25) and (39) of the Appendix, the differential cross-section (12) can be presented as

$\frac{1}{L} \frac{d \sigma(\varphi)}{d \varphi}=\bar{k}\left\{A_{+}^{2} \sin ^{2} \varphi / 2+\exp (-2 \bar{q}) \cos ^{2} \varphi / 2\right\}^{1 / 2} \times$

$\times\left\{A_{-}^{2} \sin ^{2} \varphi / 2+\exp (-2 \bar{q}) \cos ^{2} \varphi / 2\right\}^{1 / 2}$,

where $L=2 \pi \lambda^{2} l$ is a quantity having dimension of length and $A_{ \pm}=\lambda\left[K_{1}(\bar{q})-\bar{q} K_{2}(\bar{q}) / 4\right] / 2 \pm \exp (-\bar{q})$, $K_{1,2}(\bar{q})$ are the modified Bessel functions of the second kind of the order 1 and 2, respectively.

It follows from (26) that the backscattering differential cross-section $(\varphi=\pi)$ is not equal to zero and is given by the expression

$\left.\frac{1}{L} \frac{d \sigma(\varphi)}{d \varphi}\right|_{\varphi=\pi}=\bar{k}\left|\frac{\lambda^{2}}{4}\left[K_{1}(2 \bar{k})-\frac{\bar{k}}{2} K_{2}(2 \bar{k})\right]^{2}-\exp (-4 \bar{k})\right|$.

Here, we used $\bar{q}=2 \bar{k} \sin \pi / 2=2 \bar{k}$.

Formula (26) allows one to analyze the differential cross-section as a function of the scattering angle $\varphi$ and the dimensionless wave vector $\bar{k}$ that is connected with the energy of incident electrons $E$ by the relation $\bar{k}=E l / \hbar v_{\mathrm{F}}$. The case of slow electrons $(\bar{k} \ll 1, \bar{k}>\lambda)$ can be considered analytically, by using the asymptotic expansions (41) of the Appendix. With account for the leading terms, relation (26) yields

$\left.\frac{1}{L} \frac{d \sigma(\varphi)}{d \varphi}\right|_{\varphi=\pi}=\bar{k} \exp (-4 \bar{k})$.
It is necessary to note that the application of the Born approximation to the electron scattering in graphene by the nonhomogeneous magnetic field of remote nanomagnets requires that the following inequality $\hbar k \gg(e / c) A)$, which follows from (1), must be true (the magnetic field of a nanomagnet must be a small perturbation). Introducing $\bar{k}=k l$ and the typical value of the vector potential (14), (15) with account of (17), we get the inequality

$\bar{k} \gg \frac{e d}{\hbar c l} \equiv \lambda$.

We note that, for $\lambda \ll 1$, inequality (29) becomes $\bar{k}^{2} \gg \lambda^{2}$. It can be easily seen from (8) and (9), if we use the dimensionless variables.

\section{Scattering by a Magnetic Dipole Parallel to the Graphene Plane}

Next, we consider the electron scattering by remote magnetic dipoles parallel to the graphene plane. For the sake of simplicity, we consider two cases where the incident beam of electrons propagates along the magnetic dipole and transversally to it. The expressions of $V_{1,2}^{\|, \perp}(9)$ with $\mathbf{A}$ and $B_{z}$ from (15), where we set $\alpha=0, \pi / 2$, respectively, can be written in the dimensionless form as

$V_{1,2}^{\|}=\frac{\lambda}{\bar{R}^{3}}\left\{\frac{\lambda\left(1+y^{2}\right)}{\bar{R}^{3}} \mp 3 \frac{x}{\bar{R}^{2}}\right\}$,

$V_{1,2}^{\perp}=\frac{\lambda}{\bar{R}^{3}}\left\{\frac{\lambda\left(1+x^{2}\right)}{\bar{R}^{3}}+2 \bar{k} \mp 3 \frac{y}{\bar{R}^{2}}\right\}$.

With the help of these expressions, the scattering amplitudes (11) after passing to the coordinate system with the $y^{\prime}$-axis parallel to $\mathbf{q}$ like in the previous section, we obtain the integrals

$f_{1,2}^{\|}(\varphi)=-\frac{\lambda}{4} \sqrt{\frac{l}{\pi \bar{k}}} \int_{0}^{\infty} d r^{\prime} \int_{0}^{2 \pi} d \phi^{\prime} \exp \left(-i \bar{q} r^{\prime} \cos \phi^{\prime}\right) \times$
$\times\left\{\frac{\lambda\left[r^{\prime}+r^{\prime 3} \cos ^{2}\left(\phi^{\prime}-\frac{\varphi}{2}\right)\right]}{R^{\prime 6}} \mp 3 \frac{r^{\prime 2} \sin \left(\phi^{\prime}-\frac{\varphi}{2}\right)}{R^{\prime 5}}\right\}$,

$f_{1,2}^{\perp}(\varphi)=-\frac{\lambda}{4} \sqrt{\frac{l}{\pi \bar{k}}} \int_{0}^{\infty} d r^{\prime} \int_{0}^{2 \pi} d \phi^{\prime} \exp \left(-i \bar{q} r^{\prime} \cos \phi^{\prime}\right) \times$

$\times\left\{\frac{\lambda\left[r^{\prime}+r^{\prime 3} \sin ^{2}\left(\phi^{\prime}-\frac{\varphi}{2}\right)\right]}{R^{\prime 6}}+\right.$

$\left.+\frac{2 \bar{k} r^{\prime}}{R^{\prime 3}} \mp 3 \frac{r^{\prime 2} \cos \left(\phi^{\prime}-\varphi / 2\right)}{R^{\prime 5}}\right\}$.

ISSN 2071-0194. Ukr. J. Phys. 2016. Vol. 61, No. 5 
Integrals over $\varphi^{\prime}$ and $r^{\prime}$ in (32) and (33) are given in the Appendix. The final results read

$f_{1,2}^{\|}(\varphi)=-\frac{\lambda}{2} \sqrt{\frac{\pi l}{\bar{k}}}\left\{I_{\|}(\bar{q}) \mp 3 i I_{15}^{(2)}(\bar{q}) \sin \varphi / 2\right\}$,

and

$f_{1,2}^{\perp}(\varphi)=-\frac{\lambda}{2} \sqrt{\frac{\pi l}{\bar{k}}}\left\{I_{\perp}(\bar{q}) \pm 3 i I_{15}^{(2)}(\bar{q}) \cos \varphi / 2\right\}$.

Here,

$I_{\|}(\bar{q})=\lambda\left[I_{06}^{(1)}(\bar{q})-\frac{1}{\bar{q}} I_{16}^{(2)} \cos \varphi+I_{06}^{3}(\bar{q}) \cos ^{2} \varphi / 2\right]$,

and

$I_{\perp}(\bar{q})=\lambda\left[I_{06}^{(1)}(\bar{q})+\frac{1}{\bar{q}} I_{16}^{(2)}(\bar{q}) \cos (\varphi)+I_{06}^{(3)} \sin ^{2} \varphi / 2\right]+$ $+2 \bar{k} I_{03}^{(1)}(\bar{q})$.

The differential scattering cross-sections by remote magnetic dipoles parallel and perpendicular to the incident beams of electrons in graphene according to (12) and (34), (35) with usage of (40) from the Appendix are given by

$$
\begin{aligned}
& \frac{1}{L} \frac{d \sigma_{\|}(\varphi)}{d \varphi}=\bar{k}\left[\frac { \lambda ^ { 2 } } { 6 4 } \left[\bar{q} K_{2}(\bar{q}) \sin ^{2} \frac{\varphi}{2}+\right.\right. \\
& \left.\left.+K_{1}(\bar{q})(2+\cos \varphi)\right]^{2}+\exp (-2 \bar{q}) \sin ^{2} \frac{\varphi}{2}\right] \sin ^{2} \frac{\varphi}{2} \\
& \frac{1}{L} \frac{d \sigma_{\perp}(\varphi)}{d \varphi}=\bar{k}\left[\left(\frac { \lambda } { 8 } \operatorname { s i n } \frac { \varphi } { 2 } \left[\bar{q} K_{2}(\bar{q}) \cos ^{2} \frac{\varphi}{2}+\right.\right.\right. \\
& \left.\left.\left.+K_{1}(\bar{q})(2-\cos \varphi)\right]+\exp (-\bar{q})\right)^{2}+\frac{1}{4} \exp (-2 \bar{q}) \sin ^{2} \varphi\right]
\end{aligned}
$$

These formulas show that the backscattering crosssections $(\varphi=\pi, \bar{q}=2 \bar{k})$ are nonzero in both cases

$$
\begin{aligned}
& \frac{1}{L} \frac{d \sigma_{\|}(\varphi)}{d \varphi}=\bar{k}\left\{\frac{\lambda^{2}}{64}\left[2 \bar{k} K_{2}(2 \bar{k})+K_{1}(2 \bar{k})\right]^{2}+\exp (-4 \bar{k})\right\} \\
& \frac{1}{L} \frac{d \sigma_{\perp}(\varphi)}{d \varphi}=\bar{k}\left(\frac{3 \lambda}{8} K_{1}(2 \bar{k})+\exp (-2 \bar{k})\right)^{2} .
\end{aligned}
$$

For $\bar{k}>1$, the cross sections (36) are practically equal, but they are small due the asymptotic behavior (see Appendix).

Figure 2 depicts the graphs of $d \sigma(\varphi) / d \varphi$ versus the scattering angle $\varphi$ in units $L$ for a particular

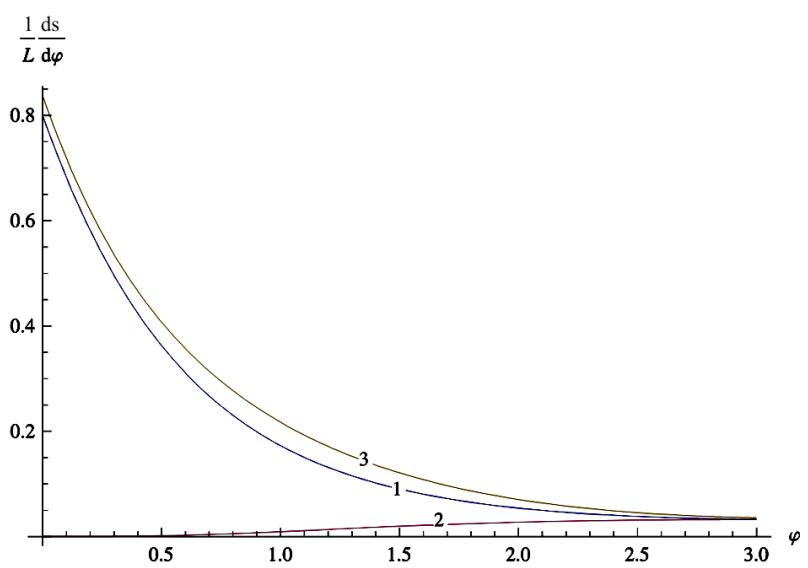

Fig. 2. Differential cross-section $d \sigma / d \varphi$ in units of $L$ versus the scattering angle $\varphi$ for $\lambda=0.1$ and $\bar{k}=0.8$. Curve 1 for the magnetic dipole moment of a nanomagnet perpendicular to the graphene plane, curve 2 for the magnetic dipole moment of a nanomagnet parallel to the incident electron beam in the graphene plane, curve 3 for the magnetic dipole moment of a nanomagnet parallel to the graphene plane and perpendicular to the incident electron beam in the graphene plane

value of $\bar{k}$ built according to (26) (curve 1) and (36) (dipole parallel to the incident electron beam curve 2, and a dipole transverse to the incident electron beam curve 3). Here, we consider $\lambda=0.1$; $\bar{k}=0.8$. Comparing curve 1 with the result obtained in [2], the differential scattering cross-section is enhanced 20 times. Curve 2 is drawn for the case where the magnetic dipole of a nanomagnet is parallel to both the graphene plane and the incident beam of electrons. As one can see from the graph, the differential cross-section is much smaller than those shown by curve 1 and 3. This indicates that if one is interested to obtain large scattering lengths, he/she has to arrange the electrons beam transverse to the direction of the dipole of the nanomagnet. One more feature of the graphs is the similarity between curve 1 and 3. One common feature of the differential cross-section graphs is that, for $\bar{k} \ll 1$, the graphs show the isotropic property. For $\bar{k} \gg 1$, it goes to zero. The pattern of our graphs and the numerical results are similar to the results obtained in [8].

\section{Transport Electron Cross-Sections}

The transport cross-section or transport scattering length, the quantity which controls the transport phe- 


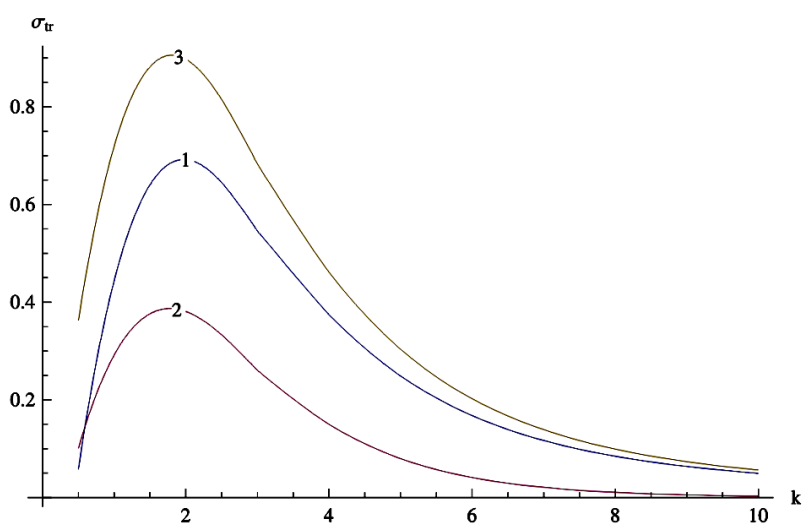

Fig. 3. Transport cross-section $\sigma$ divided by $L$ versus the dimensionless energy $\bar{k}$ for $\lambda=0.1$. Curve 1 : the magnetic dipole is transverse to the graphene plane. Curve 2: the magnetic dipole is parallel to the graphene plane and parallel to the incident beam of electrons. Curve 3: the magnetic dipole is parallel to the graphene plane and perpendicular to the incident beam of electrons. Here, we consider few leading terms of (26) and (36) and apply the extrapolation method

nomena, is given by the relation

$\sigma_{t r}=\int_{0}^{2 \pi}(1-\cos \varphi) \frac{d \sigma}{d \varphi} d \varphi$

Graphs in Fig. 3 illustrate the electron transport cross-section built in according to (38). The graphs show that the electron transport cross-section, which is directly related to the resistivity of the material is large, when the electron beams in the graphene plane are perpendicular to the suspended dipole of a nanomagnet, curve 1 and 3. One common feature of these curves is that they have maxima around $\bar{k}=1.8$ with little variation. Other numerical results show that the peaks of these maxima are shifted to left for small value of $\lambda$.

Comparing the present result with [2], it is possible to enhance the scattering length and the transport cross-section 10 to 100 times depending on the value of $\lambda$, but we have to be alert about the inequality given by (29). Our graphical results are similar to the results obtained in [9].

\section{Conclusions}

In this paper, we have studied the elastic electron scattering by remote nanomagnets transverse and parallel to the graphene plane. We have analyzed the scattering of massless Dirac fermions by a fixed magnetic dipole moment located at a height $l$ above the origin of the $2 \mathrm{D}$-graphene plane. With the help of the specially developed Born approximation, we derived the differential cross-section and the transport crosssection. The differential scattering cross-section and the transport cross-section are dominant when the magnetic dipole of a remote nanomagnet is transverse to the electron beam in the graphene plane. Our results show that one has to consider higher terms in the expansion of the potential given by (9), which has significant contribution to the scattering parameters, when the coupling parameter $\lambda$ is of the order of 0.1 to 1 . We have found that the backscattering cross-section of electrons is nonzero for all orientations.

\section{APPENDIX}

Integral entering into the electron differential cross-section by remote nanomagnets with the magnetic moment transversal to the graphene plane:

$I_{06}^{(3)}=\frac{\bar{q}}{2}\left[K_{1}(\bar{q})-\frac{\bar{q}}{4} K_{2}(\bar{q})\right]$,

$I_{05}^{(1)}=\frac{1+\bar{q}}{3} \exp (-\bar{q}), \quad I_{05}^{(3)}=\frac{2-\bar{q}}{3} \exp (-\bar{q})$,

$I_{13}^{(2)}=\exp (-\bar{q})$.

Integrals entering into the electron differential cross-section by remote nanomagnets with the magnetic moment parallel to the graphene plane and parallel or perpendicular to the incident beam of electrons:

$I_{06}^{(1)}=\frac{\bar{q}^{2}}{8} K_{2}(\bar{q}), \quad I_{26}^{(3)}=\frac{\bar{q}^{2}}{8} K_{0}(\bar{q})$,

$I_{15}^{(2)}=\frac{\bar{q}}{3} \exp (-\bar{q}), \quad I_{03}^{(1)}=\exp (-\bar{q}), \quad I_{16}^{(2)}=\frac{\bar{q}^{2}}{8} K_{1}(\bar{q})$.

The asymptotic expansions of the modified Bessel functions are given by [6]

$K_{0}(\bar{q})=-\ln \bar{q}-\gamma+\ln 2, \quad \bar{q} \rightarrow 0$,

$K_{n}(\bar{q})=2^{n-1}(n-1) ! \bar{q}^{(-n)}, n>0, \quad \bar{q} \rightarrow 0$,

$K_{n}(\bar{q})=\sqrt{\frac{\pi}{2 \bar{q}}} \exp (-\bar{q})\left\{1+\frac{4 n^{2}-1}{8 \bar{q}}+\ldots\right\}, \quad \bar{q} \gg 1$,

where $\gamma=0.5772$ is the Euler-Mascheroni constant.

1. M.I. Katsnelson, Graphene (Carbon in Two Dimensions) (Cambridge Univ. Press, New York, 2012).

2. V.N. Mal'nev, Teshome Senbeta, Yohannes Achenefe, Physica E 60, 214-219 (2014).

ISSN 2071-0194. Ukr. J. Phys. 2016. Vol. 61, No. 5 
3. M. Kabir, D.G. Kanhere, and A. Mookerjee, Phys. Rev. B 73, 075210-1-075210-4 (2006).

4. L. Liu, G.Y. Guo, C.S. Jayanthi, and S.Y. Wu, Phys. Rev. Lett. 88, 217206-1-217206-4 (2002).

5. L.D. Landau and E.M. Lifshitz, Quantum Mechanics (Nonrelativistic Theory) (Elsevier, Oxford, 1977).

6. G.B. Arfken and H.J. Weber, Mathematical Methods for Physicists (Academic Press, New York, 2005).

7. P.S. Novikov, Phys. Rev. B 245935 (2006).

8. A. Jablonski, F. Salvat, and C. J. Powell, J. Phys. Chem. Ref. Data 33, No. 2, (2004).

9. A. Zazunov, A. Kundu, A. Hţten, and R. Egger, Phys. Rev. B 82, 155431 (2010).

Received 15.06.15
I. Аченефе, Т. Сенбета, В.М. Малънев

РОЗСІЯННЯ ЕЛЕКТРОНІВ

ДАЛЕКИМИ НАНОМАГНІТАМИ

Р е з ю м е

Розглянуто пружне розсіяння електронів в графені неоднорідним магнітним полем далеких наномагнітів у рамках модифікованого борнівського наближення. Наномагніти моделюються точковими магнітними диполями, орієнтованими перпендикулярно і паралельно площині графена. Вони можуть створювати досить сильні магнітні поля без пошкодження площини графена. Знайдено в замкнутому вигляді і чисельно проаналізовано перетини розсіювання електронів. Показано, що цей механізм розсіювання містить ненульовий перетин розсіювання назад і може помітно впливати на провідність графена. 